https://helda.helsinki.fi

\title{
Aetiology of in-hospital cardiac arrest on general wards
}

\section{Tirkkonen, Joonas}

2016-10

Tirkkonen , J , Hellevuo , H , Olkkola , K T \& Hoppu , S 2016 , ' Aetiology of in-hospital cardiac arrest on general wards ' , Resuscitation , vol. 107 , pp. 19-24 . https://doi.org/10.1016/j.resuscitation.2016.0

http://hdl.handle.net/10138/229965

https://doi.org/10.1016/j.resuscitation.2016.07.007

publishedVersion

Downloaded from Helda, University of Helsinki institutional repository.

This is an electronic reprint of the original article.

This reprint may differ from the original in pagination and typographic detail.

Please cite the original version. 


\title{
Clinical paper
}

\section{Aetiology of in-hospital cardiac arrest on general wards $s^{\text {is }}$}

\author{
Joonas Tirkkonen $^{\mathrm{a}, \mathrm{b}, *, 1}$, Heidi Hellevuo ${ }^{\mathrm{c}, 1}$, Klaus T. Olkkola ${ }^{\mathrm{d}}$, Sanna Hoppu ${ }^{\mathrm{a}}$ \\ a Tampere University Hospital, Department of Intensive Care Medicine, P.O. Box 2000, FI-33521 Tampere, Finland \\ ${ }^{\mathrm{b}}$ Department of Anaesthesiology and Intensive Care Medicine, Seinäjoki Central Hospital, Finland \\ ${ }^{c}$ Department of Emergency Medicine, Tampere University Hospital, P.O. Box 2000, FI-33521 Tampere, Finland \\ ${ }^{\mathrm{d}}$ Department of Anaesthesiology, Intensive Care and Pain Medicine, University of Helsinki and Helsinki University Hospital, P.O. Box 340, FI-00029 HUS \\ Helsinki, Finland
}

\section{A R T I C L E I N F O}

\section{Article history:}

Received 23 March 2016

Received in revised form 11 June 2016

Accepted 14 July 2016

\section{Keywords:}

In-hospital cardiac arrest

IHCA

Aetiology

\begin{abstract}
A B S T R A C T
Aim: Aetiology of in-hospital cardiac arrests (IHCAs) on general wards has not been studied. We aimed to determine the underlying causes for IHCAs by the means of autopsy records and clinical judgement of the treating consultants. Furthermore, we investigated whether aetiology and preceding vital dysfunctions are associated with long-term survival.

Design and setting: Prospective observational study between 2009-2011 including 279 adult IHCA patients attended by medical emergency team in a Finnish university hospital's general wards.

Results: The median age of the patients was $72(64,80)$ years, $185(66 \%)$ were male, $178(64 \%)$ of events were monitored/witnessed, first rhythm was shockable in 42 (15\%) cases and 53 (19\%) patients survived six months. Aetiology was determined as cardiac in 141 events, 73 of which were due to acute myocardial infarction. There were 138 non-cardiac IHCAs; most common causes were pneumonia (39) and exsanguination (16). No statistical difference was observed in the incidence of objective vital dysfunctions preceding the event between the cardiac and non-cardiac groups ( $40 \%$ vs. $44 \%, p=0.448$ ). Subjective antecedents were more common in the cardiac cohort ( $47 \% \mathrm{vs.} 32 \%, p=0.022$ ), chest pain being an example ( $11 \%$ vs. $0.7 \%, p<0.001$ ). Reviewing all 279 IHCAs, only shockable primary rhythm, monitored/witnessed event and low comorbidity score were independently associated with 180-day survival. Conclusions: Cardiac aetiology underlies half of the IHCAs on general wards. Both objective and subjective antecedents are common. However, neither the cardiac aetiology nor the absence of preceding deterioration of vital signs were factors independently associated with a favourable outcome.
\end{abstract}

(c) 2016 Elsevier Ireland Ltd. All rights reserved.

\section{Introduction}

Survival to discharge after in-hospital cardiac arrest (IHCA) remains poor (10-20\%) and it has practically remained unchanged through the recent decades. ${ }^{1-4}$ Cardiac arrest outside hospital is presumed to be of cardiac origin if no other definite signs exist; IHCAs on the other hand are often preceded by derangements in vital signs and cardiac aetiology is not assumed by default. ${ }^{5,6}$ Furthermore, IHCA victims often have several comorbidities in

\footnotetext{
is A Spanish translated version of the abstract of this article appears as Appendix in the final online version at http://dx.doi.org/10.1016/j.resuscitation.2016.07.007.

* Corresponding author at: Tampere University Hospital, Department of Intensive Care Medicine, P.O. Box 2000, FI-33521 Tampere, Finland.

E-mail addresses: tirkkonen.joonas.o@student.uta.fi (J. Tirkkonen), heidi.hellevuo@fimnet.fi (H. Hellevuo),klaus.olkkola@helsinki.fi (K.T. Olkkola), sanna.hoppu@pshp.fi (S. Hoppu).

1 Equal contribution.
}

addition to the concurrent illness, making the situation even more precarious. $^{7,8}$

Data on the definite aetiology of IHCAs on hospital general wards are very limited although the epidemiological understanding could result in improved and more prompt peri- and postarrest care. Bergum et al. studied 258 IHCAs and found cardiac causes to be responsible for $60 \%$ of the IHCAs. ${ }^{9}$ Wallmuller et al. reported similarly the percentage to be 63 , and cardiac aetiology was associated with better outcome. ${ }^{10}$ However, in these thorough studies only $50 \%$ and $19 \%$ of IHCAs occurred on general wards while the rest occurred in specialized departments inside hospital like emergency departments, intensive care units and post anaesthetic care units. ${ }^{9,10}$

The aim of this study was to shed light on the aetiology and antecedents to IHCAs occurring on hospital's general wards and investigate, whether the aetiology and antecedents are associated with outcome. 


\section{Methods}

Ethics

The Ethics Committee of the Tampere University Hospital (TAYS) approved the study protocol (Approval no: R08116; clinicaltrials.gov NCT00951704). National Institute for Health and Welfare and Regional State Administrative Agencies gave their consent to analyse forensic autopsy findings. Informed consent from the patient/relatives was waived as this study was purely observational.

\section{Hospital}

TAYS is one of the five university level tertiary referral centres in Finland with 71,000 somatic admissions per year. TAYS has a closed model, mixed surgical-medical intensive care unit(ICU) with 24 beds and approximately 2000 annual admissions. A separate cardiac ICU provides care for post operative cardiothoracic patients. Excluding the ICUs, paediatric wards, post anaesthetic care units and emergency department TAYS has 750 general ward beds, $6 \%$ of which have automated monitoring capabilities.

\section{Definitions}

IHCA was defined as cessation of cardiac activity, confirmed by the absence of signs of circulation, in a hospitalized patient who had a pulse at the time of admission. ${ }^{11}$ Three outcomes were reported for IHCA patients in this study. ROSC (return of spontaneous circulation) was defined as return of a spontaneous perfusing rhythm with a palpable pulse. ${ }^{11}$ Time-fixed outcomes, being alive $24 \mathrm{~h}$ and 180 days after the initial ROSC, were also reported. The main outcome was the 180-day survival. Vital dysfunctions preceding IHCAs were defined as hospital's positive objective MET activation criteria present during a given time period before the events, and are also referred as afferent limb failures (ALFs) in the MET literature. ${ }^{12-15}$ Our hospital's objective MET criteria are presented in Table 3. Because the time period considered to be incorporated in ALF analysis is very disperse (starting from 15-60 min before the event and ending at 420-1440 min before the event), ${ }^{12-15}$ we defined the time frame in this study to be $20-720 \mathrm{~min}$. Definitions for subjective symptoms preceding IHCAs (antecedents) are also presented in Table 3. Included symptoms were based on out-ofhospital cardiac arrest studies. ${ }^{16,17}$

\section{Medical emergency team}

The medical emergency team (MET) was implemented in January 2009. It is led by an ICU physician accompanied by two ICU nurses. The MET operates 24/7, responding to both IHCAs and other medical emergencies. During the study period, MET activation rate was approximately 10 calls per 1000 hospital admissions including IHCAs and 8.4 per 1000 admissions if just medical emergencies were included.

\section{Data collection and exclusion criteria}

Data on IHCAs were routinely prospectively collected by the MET attending the resuscitation attempts during the study period of 2009-2011. Known data on comorbidities, preceding vital dysfunctions, subjective antecedents and survival were noted from patient records. Age-adjusted Charlson comorbidity index (CCI) was calculated for each patient; the total score presents the cumulative negative impact that patient's diseases and age have on favourable outcome. ${ }^{18}$
Table 1

Specific reason for in-hospital cardiac arrest.

\begin{tabular}{|c|c|}
\hline \multicolumn{2}{|l|}{ Cardiac reason for IHCA $n=141$} \\
\hline Acute myocardial infarction & 73 \\
\hline Myocardial ischaemia (no acute infarction) & $26^{\mathrm{a}}$ \\
\hline Cardiac tamponade & 5 \\
\hline Congestive heart failure & 16 \\
\hline Complication after invasive cardiac intervention & 9 \\
\hline \multicolumn{2}{|l|}{ Other } \\
\hline Dilated cardiomyopathy & 2 \\
\hline Cardiac amyloidosis & 2 \\
\hline Cor pulmonale (after chronic pulmonary embolism) & 1 \\
\hline Atrial fibrillation & 1 \\
\hline Perimyocarditis & 1 \\
\hline Aortic stenosis & 2 \\
\hline Aortic regurgitation & 1 \\
\hline Mitral regurgitation & 1 \\
\hline Ascending aortic dissection & 1 \\
\hline \multicolumn{2}{|l|}{ Non-cardiac reason for IHCA $n=138$} \\
\hline Pulmonary embolism & 12 \\
\hline \multicolumn{2}{|l|}{ Infection } \\
\hline Pneumonia & 39 \\
\hline Peritonitis & 11 \\
\hline Urogenital infection & 2 \\
\hline Erysipelas & 1 \\
\hline Pancreatitis & 3 \\
\hline Colechystitis & 2 \\
\hline Sepsis NAS & 6 \\
\hline Cirrhosis & 3 \\
\hline Renal insufficiency & 2 \\
\hline \multicolumn{2}{|l|}{ Exsanguination } \\
\hline GI tract & 10 \\
\hline Traumatic & 3 \\
\hline Airway & 1 \\
\hline Iatrogenic & 2 \\
\hline Cerebral ischaemia & 4 \\
\hline Anaphylaxis & 2 \\
\hline Hyperkalemia & 2 \\
\hline Hyponatremia & 2 \\
\hline Hematologic malignancy & 2 \\
\hline Malignant solid tumour & 7 \\
\hline Mechanical airway obstruction & 7 \\
\hline Vasovagal collapse & 1 \\
\hline Acute mesenteric ischemia & 1 \\
\hline Overdose of opioids/benzodiazepines & 2 \\
\hline Hypoxia due to chronic lung disease & 4 \\
\hline Reason unclear & 7 \\
\hline
\end{tabular}

Data are presented in numbers. IHCA, in-hospital cardiac arrest; NAS, not further specified; GI, gastrointestinal.

a In one case ischaemia was provoked by low hematocrit.

In case the resuscitation was successful or autopsy was not deemed necessary, aetiology of the cardiac arrest was classified according to the expert opinion of the consultant(s) responsible for the patient. The 'consultant's expert opinion' refers to the final conclusion of the aetiology (obtained from the patient records and/or the death certificate) after consultant(s) had assessed all relevant patient history and conducted all diagnostic tests and interventions deemed necessary; it does not refer to the first impression of the aetiology possibly recorded to the Utstein-style resuscitation form. Study personnel did not make assumptions about the aetiology.

No autopsies were conducted as part of this study protocol. Forensic autopsies must be conducted in certain situations according to the law and the cause of death investigation is led by the police. Medical autopsies are conducted if deemed necessary by the consultant responsible for the patient (e.g. the cause of death is considered uncertain). If either forensic or medical autopsy was conducted, the cause of the cardiac arrest was obtained from the autopsy records in case no ROSC was achieved or if ROSC was achieved but patient died during the following hours/day(s).

Paediatric IHCAs were not included in this study. IHCAs outside the general wards were excluded from further analyses. 
Table 2

Patient characteristics, primary rhythm and outcome.

\begin{tabular}{|c|c|c|c|}
\hline & IHCA due to cardiac aetiology $(N=141)$ & IHCA due to non-cardiac aetiology $(N=138)$ & $p$-value \\
\hline \multicolumn{4}{|l|}{ Patient characteristics } \\
\hline Age (median; $Q_{1}, Q_{3}$ ) & $74(64,82)$ & $70(63,77)$ & 0.010 \\
\hline Sex (male) & $94(67)$ & $91(66)$ & 0.898 \\
\hline Age-adjusted CCI $($ mean \pm SD) & $5.3 \pm 2.4$ & $4.8 \pm 2.7$ & 0.112 \\
\hline Arterial hypertension & $88(62)$ & $78(57)$ & 0.316 \\
\hline Coronary artery disease & $72(51)$ & $34(25)$ & $<0.001$ \\
\hline Atrial fibrillation & $44(31)$ & $35(25)$ & 0.279 \\
\hline Cardiomyopathy & $4(1.4)$ & $0(0.0)$ & 0.122 \\
\hline Chronic heart failure & $43(31)$ & $23(17)$ & 0.007 \\
\hline Aortic stenosis & $13(9.2)$ & $4(2.9)$ & 0.043 \\
\hline Peripheral artery disease & $22(16)$ & $15(11)$ & 0.244 \\
\hline Diabetes & $44(31)$ & $36(21)$ & 0.345 \\
\hline Asthma & $8(5.7)$ & $12(8.7)$ & 0.328 \\
\hline Chronic obstructive pulmonary disease & $13(9.2)$ & $8(5.8)$ & 0.279 \\
\hline Renal insufficiency & $23(16)$ & $13(9.4)$ & 0.086 \\
\hline Dementia & $5(3.5)$ & $3(2.2)$ & 0.723 \\
\hline Malignancy & $13(9.2)$ & $30(22)$ & 0.004 \\
\hline Previously healthy & $4(2.8)$ & $4(2.9)$ & 1.000 \\
\hline \multicolumn{4}{|l|}{ Primary rhythm } \\
\hline Asystole & $45(32)$ & $47(34)$ & \\
\hline Pulseless electrical activity & $51(36)$ & $78(57)$ & \\
\hline Ventricular tachycardia & $9(6.4)$ & $2(1.4)$ & $<0.001$ \\
\hline Ventricular fibrillation & $30(21)$ & $1(0.7)$ & \\
\hline Unclear & $6(4.3)$ & $10(7.2)$ & \\
\hline Monitored/witnessed arrest & $91(65)$ & $87(63)$ & 0.795 \\
\hline \multicolumn{4}{|l|}{ Aetiology determination and outcome } \\
\hline Autopsy conducted & $81(57)$ & $72(52)$ & 0.376 \\
\hline Clinical judgement & $60(43)$ & $66(48)$ & \\
\hline ROSC & $72(51)$ & $68(49)$ & 0.765 \\
\hline Alive at $24 \mathrm{~h}$ & $54(38)$ & $60(43)$ & 0.379 \\
\hline Alive at 180 days & $29(21)$ & $24(17)$ & 0.499 \\
\hline
\end{tabular}

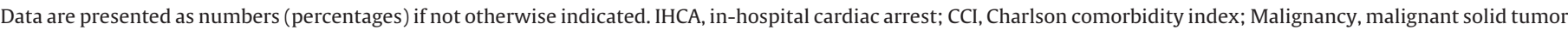
or hematologic malignancy; ROSC, return of spontaneous circulation.

Table 3

Comparison of antecedents to IHCAs related to cardiac vs. non-cardiac aetiology.

\begin{tabular}{|c|c|c|c|}
\hline & IHCA due to cardiac aetiology $(N=141)$ & IHCA due to non-cardiac aetiology $(N=138)$ & $p$-value \\
\hline \multicolumn{4}{|c|}{ Documented objective MET activation criteria (20-720 min before the IHCA) } \\
\hline Respiratory rate ( $<5$ or $>24 / \mathrm{min}$ ) & $13(9.2)$ & $17(12)$ & 0.403 \\
\hline Peripheral arteriolar $\mathrm{O}_{2}$ saturation $(<90 \%)$ & $29(21)$ & $30(22)$ & 0.811 \\
\hline Heart rate $(<40$ or $>140 / \mathrm{min})$ & $12(8.5)$ & $18(13)$ & 0.222 \\
\hline Systolic blood pressure (<90 mmHg) & $16(11)$ & $8(5.8)$ & 0.222 \\
\hline Any objective criteria & $56(40)$ & $61(44)$ & 0.448 \\
\hline Multiple objective criteria & $13(9.2)$ & $11(8.0)$ & 0.710 \\
\hline \multicolumn{4}{|c|}{ Recorded subjective antecedents (20-720 min before the IHCA) } \\
\hline Respiratory distress & $42(30)$ & $31(23)$ & 0.164 \\
\hline Chest pain & $15(11)$ & $1(0.7)$ & $<0.001$ \\
\hline Arrhythmias & $2(1.4)$ & $1(0.7)$ & 0.574 \\
\hline Upper abdominal pain & $3(2.1)$ & $4(2.9)$ & 0.681 \\
\hline Back pain & $2(1.4)$ & $2(1.4)$ & 0.983 \\
\hline 'Cold sweat' & $5(3.5)$ & $5(3.6)$ & 0.972 \\
\hline Decrease in the level of consciousness & $8(5.7)$ & $16(12)$ & 0.078 \\
\hline Any subjective antecedent & $66(47)$ & $44(32)$ & 0.022 \\
\hline Multiple subjective antecedents & $11(7.8)$ & $12(8.7)$ & 0.786 \\
\hline
\end{tabular}

Data are presented as numbers (percentages). IHCA, in-hospital cardiac arrest; MET, medical emergency team.

\section{Statistical analysis}

Data are presented as numbers (percentages) if not otherwise indicated. The chi-square test, Student's $t$-test, Fisher's exact test and Mann-Whitney $U$ test were used for comparisons between groups as appropriate. Multivariate logistic regression was applied with 'enter' model and the Hosmer-Lemeshow test was conducted to report the goodness-of-fit of the model. Tests were two-sided; $p<0.05$ was considered significant and 95\% confidence intervals were reported where appropriate. SPSS version 20 for Windows (SPSS Inc., Chicago, IL, USA) was used.

\section{Results}

\section{Study population}

Fig. 1 presents the excluded cases and the final cohort of 279 general ward patients attended by the MET because of an IHCA. Median age was $72(64,80)$ years, $185(66 \%)$ were male and mean age-adjusted $\mathrm{CCI}$ was $5.0 \pm 2.6$. Primary rhythm was shockable (VT/VF) in $42(15 \%)$ of the patients, ROSC was achieved in 140 (50\%) cases and $114(41 \%)$ patients survived the first $24 \mathrm{~h}$. After six months, 53 (19\%) patients were alive. 


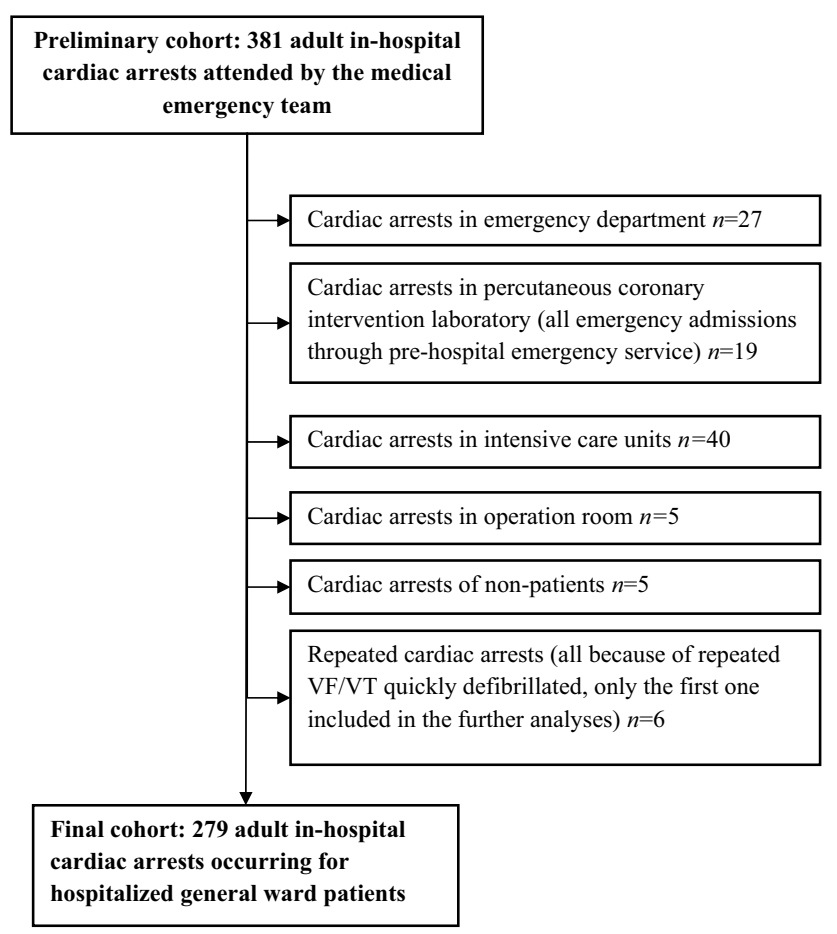

Fig. 1. Final cohort with the excluded cardiac arrests inside hospital during the study period. VF, ventricular fibrillation; VT, venticular tachycardia.

\section{Aetiology}

An autopsy was conducted in 153 (55\%) patients, 84 of which were forensic autopsies and 69 medical autopsies. For the remaining 126 (45\%) patients, the reason for IHCA was clinically assessed by the treating consultant(s); in 30 of these cases ROSC was not achieved and thus no further clinical or laboratory tests were conducted. Fig. 2 presents the determination of IHCA aetiology either as 'cardiac' or 'non-cardiac' and Table 1 lists the specific underlying aetiologies in both sub cohorts. Among patients with cardiac aetiology $(n=141,51 \%)$, acute myocardial infarction was the most common cause for IHCA. Infections were the most prevalent sub group in 'non-cardiac' category. The method for aetiology determination (autopsy vs. clinical judgement) was not associated with the determined aetiology (aetiology was determined as 'cardiac' among $53 \%$ of the autopsied patients vs. $48 \%$ among the non-autopsied patients $(p=0.376))$.

Cardiac vs. non-cardiac aetiology of IHCAs-characteristics and antecedents

Patients in the 'cardiac' and 'non-cardiac' cohorts had similar distribution of gender and cumulative comorbidity, but the 'cardiac' cohort was older (Table 2). Regarding the individual comorbidities, coronary artery disease, chronic heart failure and aortic stenosis were more prevalent in the 'cardiac' cohort. Solid or hematologic malignancies were more common among the 'non-cardiac' IHCA patients. Shockable rhythms were more common in the cardiac cohort, but non-shockable rhythms prevailed in both subcohorts. There were no differences in survival between the cohorts.

Altogether 117 (42\%) IHCA patients had had positive objective MET activation criteria 20-720 min before the event, but there were differences between the 'cardiac' or 'non-cardiac' groups (Table 3). However, the overall incidence of subjective antecedents was higher in the 'cardiac' cohort, and from the individual antecedents 15 patients in the 'cardiac' cohort had had chest pain while only one had complained this symptom in the 'non-cardiac' cohort.
Table 4

Multivariate logistic regression analysis of factors independently associated with 180-day survival.

\begin{tabular}{lllr}
\hline & \multicolumn{2}{l}{ Multivariate analysis } \\
\cline { 2 - 4 } & Odds ratio & $95 \% \mathrm{CI}$ & $p$-value \\
\hline Shockable rhythm (VT/VF) & 8.87 & $3.54-22.2$ & $<0.001$ \\
Monitored/witnessed IHCA & 2.46 & $1.13-5.36$ & 0.024 \\
Age-adjusted CCI & 0.86 & $0.75-0.99$ & 0.041 \\
Any subjective antecedent & 0.54 & $0.25-1.16$ & 0.113 \\
Cardiac aetiology & 0.59 & $0.26-1.33$ & 0.202 \\
Any objective MET criteria & 0.70 & $0.34-1.43$ & 0.324 \\
Sex (male) & 0.81 & $0.40-1.65$ & 0.563 \\
\hline
\end{tabular}

CI, confidence interval; VT, ventricular fibrillation; VF, ventricular tachycardia; IHCA in-hospital cardiac arrest; $\mathrm{CCI}$, Charlson comorbidity index; MET, medical emergency team.

Shockable primary rhythm, monitored/witnessed IHCA and lower age-adjusted CCI were independently associated with 180day survival, while cardiac aetiology and antecedent-free event were not (Table 4). The Hosmer-Lemeshow Chi-square test for multivariate logistic regression model was $4.44(p=0.816)$ indicating a good fit of the model.

\section{Discussion}

This study has two main findings. First, half of the IHCAs on hospital general wards were of cardiac origin and these IHCAs were more commonly preceded by subjective antecedents, especially chest pain, as compared to IHCAs of non-cardiac origin. Second, the multivariate logistic regression model demonstrated, that it is not the aetiology but low comorbidity, witnessed/monitored arrest and shockable primary rhythm that are independently associated with better long-term survival. To the best of our knowledge this is the first study to investigate the adjusted association between the aetiology of IHCA and outcome.

We investigated the aetiology of IHCAs on general wards, where continuous monitoring and staff resources are limited as compared to, for example, ICUs and emergency departments. Cardiac causes were still more common than non-cardiac causes, however approximately $10 \%$ less prevalent than reported in studies including all hospital departments. ${ }^{9,10}$ Of the specific causes of IHCAs, acute myocardial infarction and myocardial ischaemia without later findings of infarction together accounted for $35 \%$ of the arrests. This finding is of utmost importance, considering that only $15 \%$ of all general ward IHCAs had a shockable primary rhythm. Congestive heart failure progressing to IHCA was the second largest individual reason for arrest within the cardiac aetiology group. Following pneumonia, exsanguination and pulmonary embolism were the second and third most common individual aetiologies for IHCAs in the 'non-cardiac group'. Aetiology of altogether 112 IHCAs could be directly classified according to the '4H4T'-rule, ${ }^{6}$ even though excluding infections which potentially caused IHCAs due to hypovolaemia/hypoxia as well. This observation underlines the importance of including '4H4T'-rule in the advanced life support algorithm. ${ }^{6}$

Two thirds of the general ward IHCAs in our study were witnessed/observed. This occurrence has previously been associated with better outcome and was also confirmed in the present study. ${ }^{19,20}$ In many IHCA studies the proportion of witnessed/observed cardiac arrests has been as high as 85-92\% and it has been discussed, that previous IHCA studies represent poorly the actual patient population on general wards because of the high proportion of ICU/emergency department/cardiac care unit patients. ${ }^{9,20,21}$ Brindley et al. excluded patients allocated to 'critical care units' and found that $58 \%$ of the IHCAs were witnessed/observed, which is in line with our findings. ${ }^{19}$ Still, two 


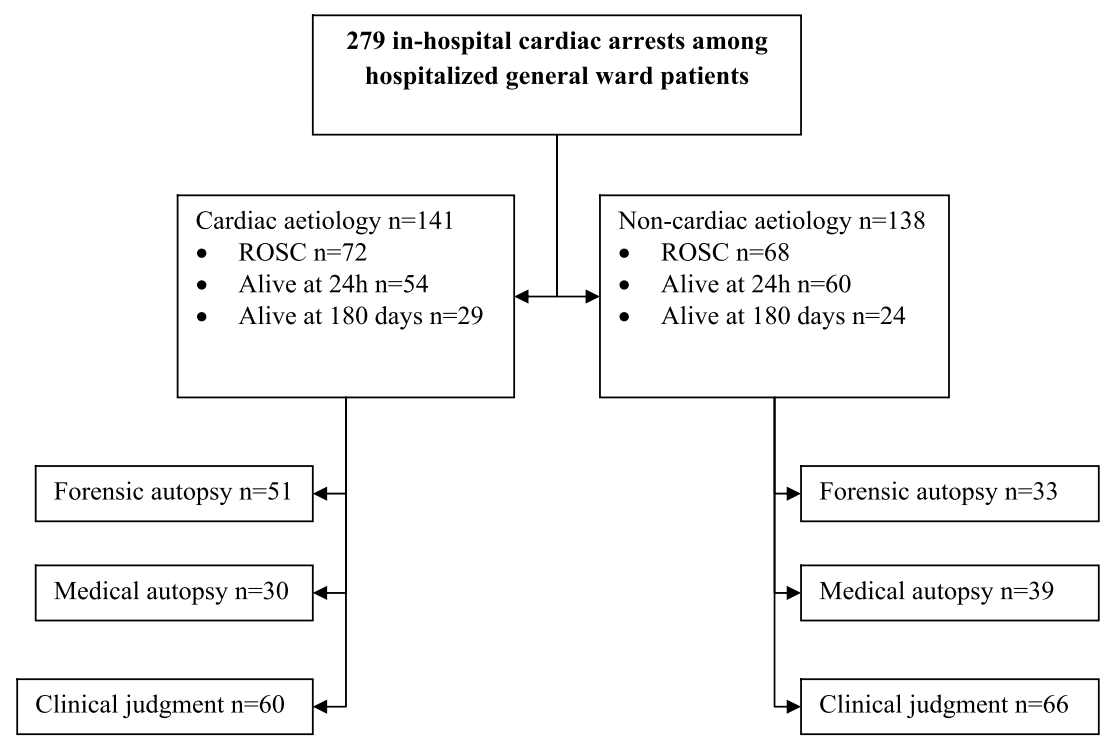

Fig. 2. Survival and methodology for determination of the aetiology among the study population. ROSC, return of spontaneous circulation.

thirds of patients experiencing a witnessed/observed cardiac arrest on general wards is rather high. However, today many hospitals have rapid response systems, ${ }^{22}$ and although we did not record the exact number, there were several cases of IHCAs where the arrest occurred during MET review and thus the arrest was naturally 'monitored and observed'.

Both vital dysfunctions and subjective antecedents were common before IHCAs, despite our hospital had implemented MET at the time this study was initiated. Similar findings have been observed in Australia: implementing a rapid response system did not automatically eliminate the intervention delays in patient care. $^{23}$ It is pivotal to disengage these afferent limb failures. ${ }^{12-15}$ Regardless of the fact that in our study these antecedents were not independently associated with worse outcome, some IHCAs could have been avoided either by appropriate interventions or ethically sound treatment limitations.

Low comorbidity, witnessed/monitored arrest and shockable primary rhythm were independently associated with six months' survival in a multivariate regression model. These pre- and periarrest factors have previously been reported to be associated with hospital- or short-term survival in univariate or multivariate analyses. 7,10,20,21,24 Our study also confirmed that these variables are associated with long-term survival, even when adjusted for gender and, for the first time, for preceding derangements and aetiology. One could have suspected that cardiac aetiology would have been associated with improved outcome, but this was not the case even with the other outcomes (ROSC, $24 \mathrm{~h}$ survival). Wallmuller et al. found cardiac aetiology to be associated with survival in a univariate analysis. ${ }^{10}$ Naturally 'cardiac aetiology' depends on how the causes are classified, but in this study the classification was quite comparable to our study. ${ }^{10}$ On the other hand, we studied the IHCAs on general wards, while Wallmuller et al. studied IHCAs mainly at the ICU of the emergency department. Our results show that if an IHCA occurs on general wards, immediate CPR (cardiopulmonary resuscitation) and shockable rhythm are the key factors for survival while comorbidities reduce probability of desired outcome.

This study has several limitations. First, as a single centre study our results may not apply in different health care settings or with distinct patient populations. Second, we could not include some factors known to be associated with at least short term survival in our multivariate analysis (e.g. adequate compression depth during $\mathrm{CPR}^{25}$ ). Third, in $11 \%$ of the cases no ROSC was achieved but autopsy was not deemed necessary; thus the consultant(s) aetiology determination relied only on patient history and pre- and periarrest factors. Fourth, the time frame of $20-720 \mathrm{~min}$ for preceding vital dysfunctions and subjective antecedents simplifies deteriorations of different durations, although it enables feasible utilization of these factors in multivariate logistic regression model and this same methodology is used in MET studies. ${ }^{12-15}$ These limitations should be weighed against the strengths of this study. Aetiology was determined by autopsy for $55 \%$ of the patients. This percentage is exceptionally high as compared to other aetiologycal studies and autopsy after an unsuccessful resuscitation attempt has been suggested as 'golden standard' for studies on the cause of cardiac arrest. ${ }^{26,27}$ We used age-adjusted charlson comorbidity index as a continuous variable to take into consideration the possible negative effect of cumulative age-related comorbidity in our analyses. ${ }^{24}$ Third, despite a single centre design, TAYS is one of the five tertiary referral centres in Finland providing the most advanced care and the general wards represent all major specialties and our study population was very heterogeneous.

\section{Conclusions}

In-hospital cardiac arrests on general wards are caused by cardiac reasons in half of the cases. Both cardiac and non-cardiac IHCAs are often preceded by vital dysfunctions and subjective antecedents. Subjective antecedents, such as 'chest pain', are more common among IHCAs of cardiac aetiology. In case of an IHCA on a general ward of the hospital, low comorbidity, witnessed/monitored arrest and shockable primary rhythm are factors independently associated with better long-term survival.

\section{Conflict of interest statement}

Authors declare no conflicts of interests.

\section{References}

1. Hershey CO, Fisher L. Why outcome of cardiopulmonary resuscitation in general wards is poor. Lancet 1982;8262:31-4.

2. Peberdy MA, Kaye W, Ornato JP, et al. Cardiopulmonary resuscitation of adults in the hospital: a report of 14720 cardiac arrests from the National Registry of Cardiopulmonary Resuscitation. Resuscitation 2003;58:297-308. 
3. Sandroni C, Nolan J, Cavallaro F, Antonelli M. In-hospital cardiac arrest: incidence, prognosis and possible measures to improve survival. Intensive Care Med 2007; 33:237-45.

4. Nolan J, Soar J, Smith GB, et al. Incidence and outcome of in-hospital cardiac arrest in the United Kingdom National Cardiac Arrest Audit. Resuscitation 2014;85:987-92.

5. Perkins GD, Handley AJ, Koster RW, et al. European Resuscitation Council Guidelines for Resuscitation 2015: section 2: adult basic life support and automated external defibrillation. Resuscitation 2015;95:81-99.

6. Soar J, Nolan JP, Böttiger BW, et al. European Resuscitation Council Guidelines for Resuscitation 2015: section 3: adult advanced life support. Resuscitation 2015;95:100-47.

7. Nadkarni VM, Larkin GL, Peberdy MA, et al. First documented rhythm and clinical outcome from in-hospital cardiac arrest among children and adults. JAMA 2006:295:50-7.

8. Nurmi J, Harjola VP, Nolan J, Castrén M. Observations and warning signs prior to cardiac arrest: should a medical emergency team intervene earlier. Acta Anaesthesiol Scand 2005:49:702-6.

9. Bergum D, Nordseth T, Mjølstad OC, Skogvoll E, Haugen BO. Causes of in-hospital cardiac arrest-incidences and rate of recognition. Resuscitation 2015;87:63-8.

10. Wallmuller C, Meron G, Kurkciyan I, Schober A, Stratil P, Sterz F. Causes of in-hospital cardiac arrest and influence on outcome. Resuscitation 2012;83:1206-11.

11. Jacobs I, Nadkarni V, Bahr J, et al. Cardiac arrest and cardiopulmonary resuscitation outcome reports: update and simplification of the Utstein templates for resuscitation registries. A statement for healthcare professionals from a task force of the international liaison committee on resuscitation (American Heart Association, European Resuscitation Council, Australian Resuscitation Council, New Zealand Resuscitation Council, Heart and Stroke Foundation of Canada, InterAmerican Heart Foundation, Resuscitation Council of Southern Africa). Resuscitation 2004;63:233-49.

12. Calzavacca P, Licari E, Tee A, et al. A prospective study of factors influencing the outcome of patients after a Medical Emergency Team review. Intensive Care Med 2008;34:2112-6.

13. Calzavacca P, Licari E, Tee A, et al. The impact of rapid response system on delayed emergency team activation patient characteristics and outcomes-a follow-up study. Resuscitation 2010;81:31-5.

14. Boniatti MM, Azzolini N, Viana MV, et al. Delayed medical emergency team calls and associated outcomes. Crit Care Med 2014:42:26-30.
15. Barwise A, Thongprayoon C, Gajic O, Jensen J, Herasevich V, Pickering BW Delayed rapid response team activation is associated with increased hospital mortality, morbidity, and length of stay in a tertiary care institution. Crit Care Med 2016;44:54-63.

16. Höglund H, Jansson JH, Forslund AS, Lundblad D. Prodromal symptoms and health care consumption prior to out-of-hospital cardiac arrest in patients without previously known ischaemic heart disease. Resuscitation 2014;85:864-8.

17. Nishiyama C, Iwami T, Kawamura T, et al. Prodromal symptoms of out-ofhospital cardiac arrests: a report from a large-scale population-based cohort study. Resuscitation 2013;84:558-63.

18. Charlson M, Szatrowski TP, Peterson J, Gold J. Validation of a combined comorbidity index. J Clin Epidemiol 1994;47:1245-51.

19. Brindley PG, Markland DM, Mayers I, Kutsogiannis DJ. Predictors of survival following in-hospital adult cardiopulmonary resuscitation. CMAJ 2002:167:343-8.

20. Brady WJ, Gurka KK, Mehring B, Peberdy MA, O'Connor RE, American Heart Association's Get with the Guidelines (formerly, NRCPR) Investigators. In-hospital cardiac arrest: impact of monitoring and witnessed event on patient survival and neurologic status at hospital discharge. Resuscitation 2011:82:845-52

21. Herlitz J, Bång A, Aune S, Ekström L, Lundström G, Holmberg S. Characteristics and outcome among patients suffering in-hospital cardiac arrest in monitored and non-monitored areas. Resuscitation 2001;48:125-35.

22. Winters BD, Weaver SJ, Pfoh ER, Yang T, Pham JC, Dy SM. Rapid-response systems as a patient safety strategy: a systematic review. Ann Intern Med 2013;158:417-25.

23. Vetro J, Natarajan DK, Mercer I, et al. Antecedents to cardiac arrests in a hospital equipped with a medical emergency team. Crit Care Resusc 2011;13:162-6.

24. Piscator E, Hedberg P, Göransson K, Djärv T. Survival after in-hospital cardiac arrest is highly associated with the age-combined Charlson co-morbidity Index in a cohort study from a two-site Swedish University hospital. Resuscitation 2016;99:79-83.

25. Stiell IG, Brown SP, Christenson J, et al. What is the role of chest compression depth during out-of-hospital cardiac arrest resuscitation. Crit Care Med 2012;40:1192-8

26. Silfvast T. Cause of death in unsuccessful prehospital resuscitation. J Intern Med 1991;229:331-5.

27. Virkkunen I, Paasio L, Ryynänen S, et al. Pulseless electrical activity and unsuccessful out-of-hospital resuscitation: what is the cause of death. Resuscitation 2008;77:207-10 